\title{
An Adaptive Edge Detection for Mycosis Fungoides Skin Disease
}

\author{
G. Ramakrishna \\ Asst. Prof in IT \\ SRESCOE \\ kopargaon- \\ Maharastra
}

\author{
Debabrata Swain \\ Asst. Prof in IT \\ SRESCOE \\ kopargaon- \\ Maharastra
}

\author{
Jore Sandeep S \\ Asst. Prof in IT \\ SRESCOE \\ kopargaon- \\ Maharastra
}

\author{
K. Gopi Krishna \\ Senior Associate \\ Genpact - G.E \\ Hyderabad
}

\begin{abstract}
Edge is an important feature of an image and provides important image information that can be used for image interpretation. Many techniques of edge detection have been developed. This paper proposes an adaptive edge detection to accurately detect the most affected part of Mycosis Fungoides skin disease image. In this paper, this new method overcomes some common disadvantages of some traditional existing edge detection operators by properly detecting the boundary region of most affected part of skin disease. This proposed method compared with existing edge detection operators.
\end{abstract}

\section{Keywords}

Robert operator, Prewitt operator, Sobel operator, zero crossing detector, laplacian.

\section{INTRODUCTION}

Edge detection is a terminology in image processing that refers to algorithm which aim at identifying edges in an image. Edges are significant local changes of intensity in an image. An edge detector accepts a digital image as input and produces an edge map as output. Classical methods of edge detection involve convolving the image with an operator (a 2D filter), it is constructed to be sensitive to large gradients in the image while returning values of zero in uniform regions. Edges can be modeled according to their intensity profiles[1].

Step edge: intensity of image abruptly changes from one value to one side of discontinuity to a different value on opposite side.

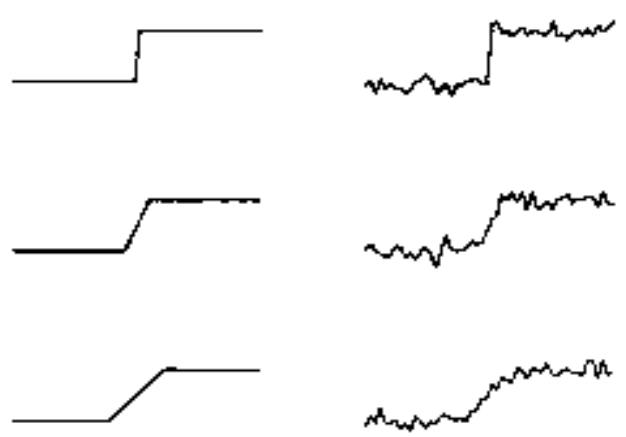

Fig 1.a) intensity of ideal images b) intensity of noisy images
Ridge edge: intensity of image abruptly changes value but returns to starting value after short distance

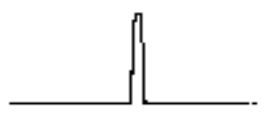

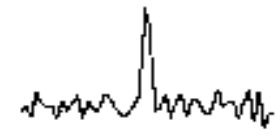
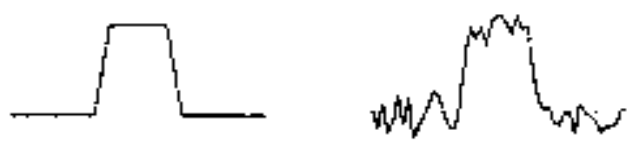

Fig 2. a) intensity of ideal images b) intensity of noisy images

Roof edge: Here intensity change is not instantaneous occur over a finite distance .
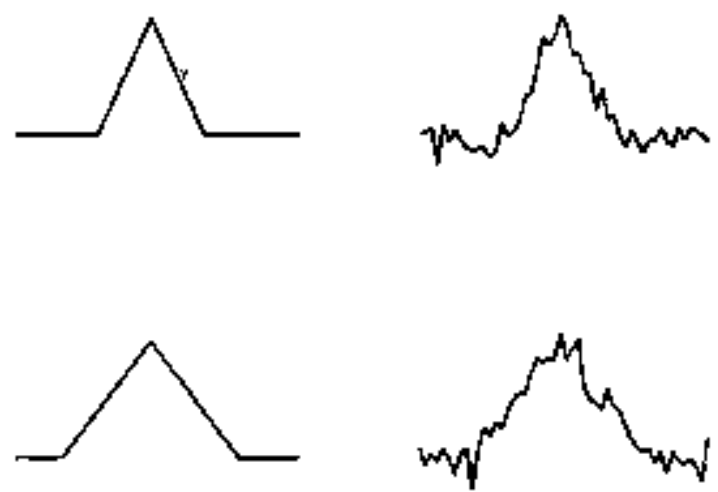

Fig 3. a) intensity of ideal images b)intensity of noisy images

\subsection{Steps of edge detection:}

a) Smoothing : suppress noise without destroying the true edges.

b) Enhancement : enhance the quality of the edges in the image using filter.

c) Detection : determine edge pixels discarded as noise.

d) Localization : Locate the edge accurately, estimate edge orientation[2]. 
There are many ways to perform edge detection. However, the majority of different methods may be grouped into two categories:

\subsection{Gradient}

In this method look for the maximum and minimum in the first derivative of the image to detect the edges.

The gradient is a vector, whose components measure how rapidly pixel values are changing with distance in the $\mathrm{x}$ and $\mathrm{y}$ directions.

For a continuous two dimensional function gradient is defined as

$$
\begin{aligned}
& G[f(x, y)]=\left[\begin{array}{l}
G x \\
G y
\end{array}\right]=\left[\begin{array}{l}
\frac{\partial f}{\partial x} \\
\frac{\partial f}{\partial y}
\end{array}\right] \\
& |G|=\sqrt{G x^{2}+G y^{2}} \approx|G x|+|G y| \\
& \theta=\tan ^{-1}\left(\frac{G y}{G x}\right)
\end{aligned}
$$

\subsection{Laplacian}

A very popular second order operator is the Laplacian operator.

It is also possible to use second order derivatives to detect edges.

The edges can be detected by zero crossings in second derivative of the image. Laplacian method searches for zero crossings to detect edges.

Zero crossing of the second derivative of a function indicates the presence of maxima.

$$
L(x, y)=\frac{\partial^{2} I}{\partial x^{2}}+\frac{\partial^{2} I}{\partial y^{2}}
$$

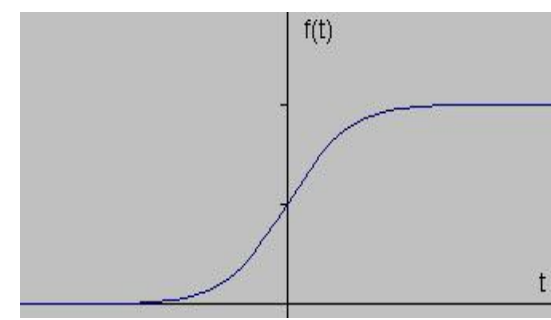

Fig 4. a) signal with an edge

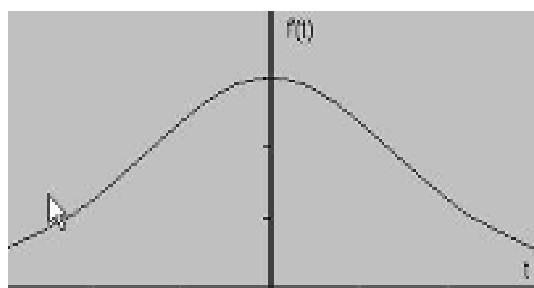

Fig 4.b) first derivative of signal w.r.t $t$

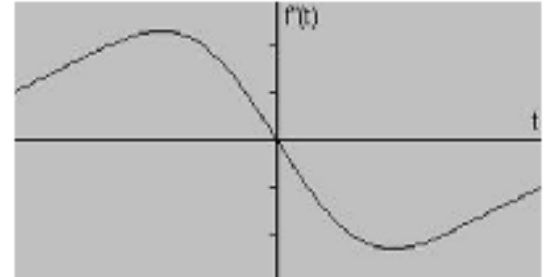

Fig 4.c) second derivative of signal w.r.t

The Laplace operator is a popular operator approximating the second derivative which gives the gradient magnitude [3].

\subsection{Advantages and Applications of Edge detection}

1) Edge detection is to discover the information about the shapes and the reflectance or transmittance in an image. It is one of the fundamental steps in image processing, image analysis, image pattern recognition, and computer vision, as well as in human vision.

2) Its Application area reaches from astronomy to medicine where isolation of objects focused on from the unwanted background is of great interest.

3) It has also found applications for photogrammetric purposes.

\section{LITERATURE SURVEY}

\subsection{D-Convolution}

The outcome of convolution operation at each pixel is the sum of multiplications between neighboring pixels of the point in the image and pixels in a kernel.The output pixel value is the weighted sum of the input pixels within the window where the weights are the values of the filter assigned to every pixel of the window itself. The window with its weights is called the convolution kernel [4].

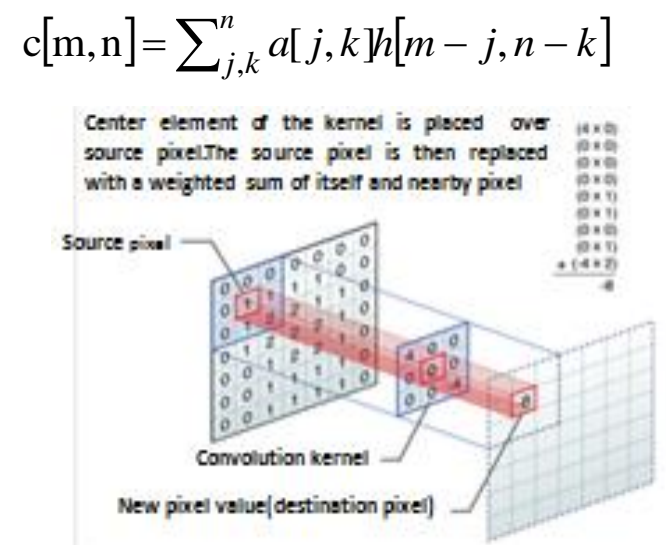

Fig 5: A single location in a 2-D convolution

\subsection{Zero crossing detector}

A more modern view to handling the problem of edges in noisy images is to use the zero crossings generated in the Laplacian of an image.Zero crossings always lie on closed contours, then the output from the zero crossing detector is usually a binary image with single pixel thickness lines showing the positions of the zero crossing points[5]. 


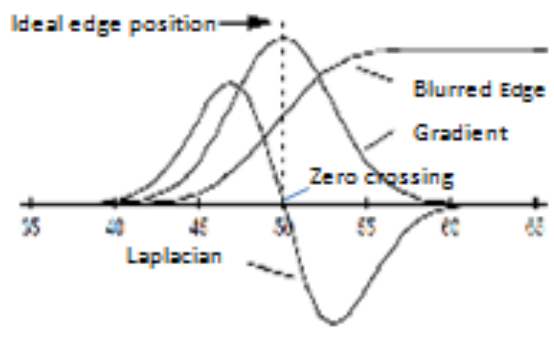

Fig 6: edge finding based on zero crossing as determined by second derivative,the Laplacian

\subsection{Histogram Equalization}

Normally,images whose pixels having the entire range of intensity levels tend to be distributed uniform will have an high contrast

To achieve this effect ,develop a transformation function based on the histogram of the input image[6].

Assume continuous intensity values with intensity $r$, where $r$ in the range $[0, \mathrm{~L}-1]$

consider transform of the form $s=T(r), 0 \leq r \leq L-1$

Here practically implemented a digital approximation of the cumulative distribution function.

$$
p_{\mathrm{r}}\left(r_{k}\right)=\frac{n_{k}}{n}
$$

Where $n_{k}$ represents how many pixels have gray level $r_{k}$

Each input gray level $r_{k}$ is mapped to an output gray level $s_{k}$ by:

$$
s_{k}=T\left(r_{k}\right)=\sum_{j=0}^{k} p_{r}\left(r_{j}\right)=\sum_{j=0}^{k} \frac{n_{j}}{n}
$$

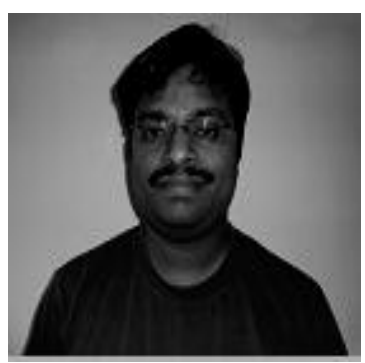

Fig 7. a) Original image before histogram Equalization

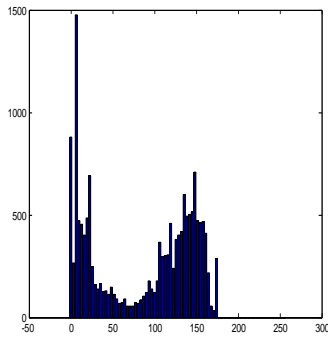

b) Histogram for original image

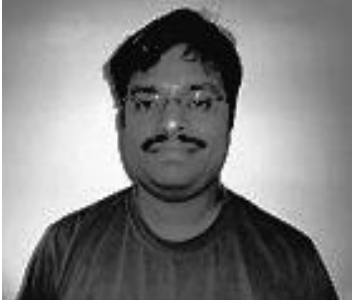

Fig 8. a) Original image after histogram Equalization

\subsection{Edge Detection Techniques}

\subsubsection{Sobel Operator}

This operator performs 2-D spatial gradient measurement on images .It consists of $3 \times 3$ convolution kernels as shown in below. One kernel is simply the other rotated by $90^{\circ}$.Here, one kernel estimating gradient in $\mathrm{x}$-direction and other estimating in y-direction.

$G y=\left[\begin{array}{ccc}1 & 2 & 1 \\ 0 & 0 & 0 \\ -1 & -2 & -1\end{array}\right]$

$$
G x=\left[\begin{array}{lll}
-1 & 0 & 1 \\
-2 & 0 & 2 \\
-1 & 0 & 1
\end{array}\right]
$$

The kernels can be applied separately to input image,to produce separate measurements of gradient component in each orientation

The gradient magnitude is given by:

$|G|=\sqrt{G x^{2}+G y^{2}} \approx|G x|+|G y|$

which is much faster to compute.

The angle of orientation of the edge giving rise to the spatial gradient is given by:

$$
\theta=\tan ^{-1}\left(\frac{G y}{G x}\right)
$$

Even this edge detector sensitive to noise,it represents them as edges.This operator is recommends for edge detection

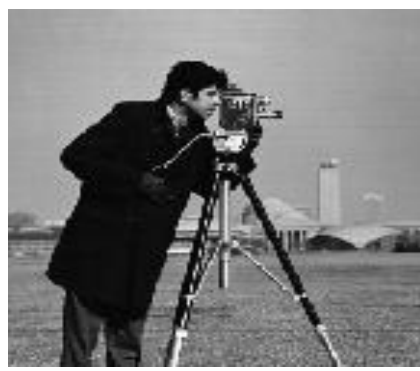

Fig 9.a) Original image 


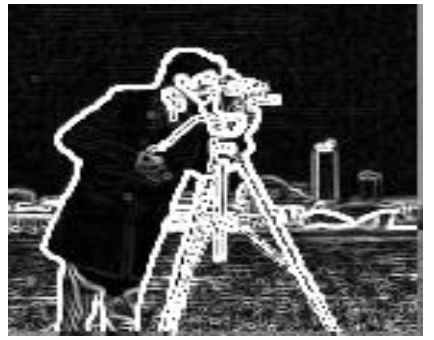

Fig 9.b)Edge detection for original image by sobel operator

\subsubsection{Robert's operator}

The Roberts operator performs a simple, quick to compute, 2D spatial gradient measurement on an image.

This operator marks edge pointers only.Work best with binary images.

The operator consists of a pair of $2 \times 2$ convolution kernels as shown below. One kernel is simply the other rotated by $90^{\circ}$. This is very similar to the Sobel operator.

$$
G x=\left[\begin{array}{cc}
1 & 0 \\
0 & -1
\end{array}\right] \quad G y=\left[\begin{array}{cc}
0 & -1 \\
1 & 0
\end{array}\right]
$$

These kernels are designed to respond maximally to edges running at $45^{\circ}$ to the pixel grid, one kernel for each of the two perpendicular orientations. The kernels can be applied separately to the input image, to produce separate measurements of the gradient component in each orientation . The gradient magnitude is given by:

$$
|G|=\sqrt{G x^{2}+G y^{2}} \approx|G x|+|G y|
$$

which is much faster to compute.

The angle of orientation of the edge giving rise to the spatial gradient (relative to the pixel grid orientation) is given by:

$$
\theta=\tan ^{-1}\left(\frac{G y}{G x}\right)-3 \prod / 4
$$

Primary disadvantage:

i) High sensitivity to noise

ii) Few pixels are used to approximate the gradient

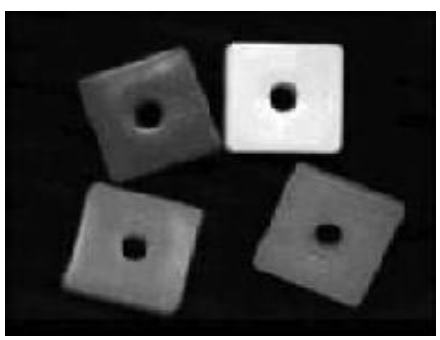

Fig 10.a) original image

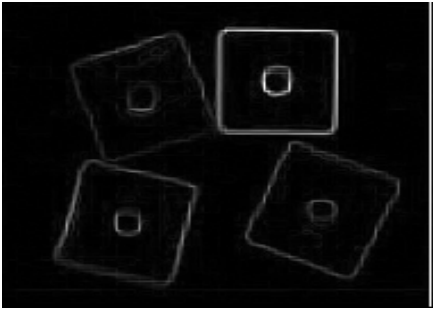

Fig 10. b) Edge detection for original image by Robert operator

\subsubsection{Prewitt's operator}

Prewitt operator is similar to the Sobel operator and is used for detecting vertical and horizontal edges in images by using kernels given below [7-8]

$$
G y=\left[\begin{array}{ccc}
1 & 1 & 1 \\
0 & 0 & 0 \\
-1 & -1 & -1
\end{array}\right] \quad G x=\left[\begin{array}{ccc}
-1 & 0 & 1 \\
-1 & 0 & 1 \\
-1 & 0 & 1
\end{array}\right]
$$

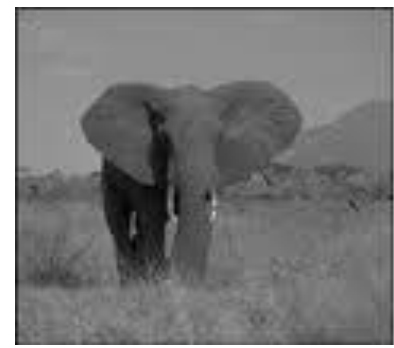

Fig 11.a) Original image

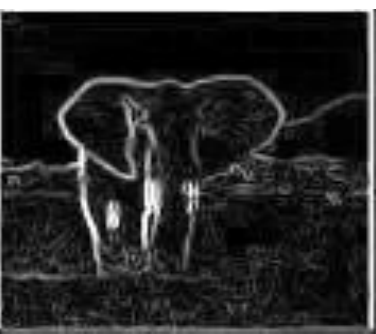

Fig 11. b) Edge detection for original image by Prewitt operator

\section{PROPOSED ALGORITHM}

Here, before implementing the algorithm. Read the input image. Convert that image into binary image. Apply convolution operation on binary image. We get the intermediate resultant image 1.

In Other side, apply Histogram Equalization to input image to get resultant image2.After that zero crossing detector is applied to get intermediate resultant image3.Perform OR operation between resultant image 2 and image 3 to yield intermediate resultant image4.Finally perform Addition. 
STEPS:

1) Read the input image and convert into binary image

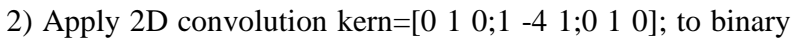
image to get Resultant Intermediate Image 1

3)To the same input binary image apply Histogram Equalization to get Resultant Intermediate Image2
4) Laplacian operator using zero crossing detector is applied for Image 2 to get intermediate resultant image 3 .

5) Perform OR operation between resultant image 2 and image 3 to get intermediate resultant image 4

6) Finally perform addition operation between image1 and image4 to get the final resultant image.

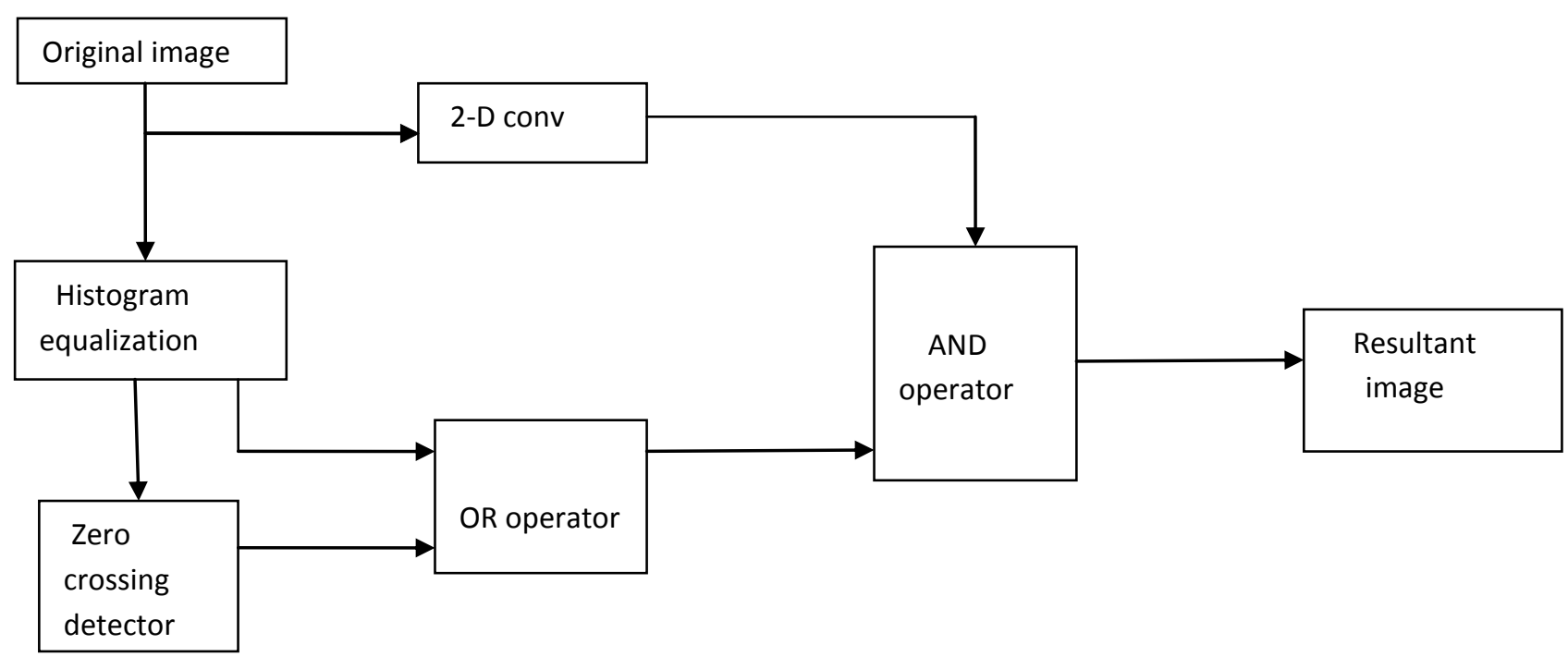

Fig 12: Diagramatic representation of Proposed Technique

\section{EXPERIMENT RESULTS AND COMPARISIONS}
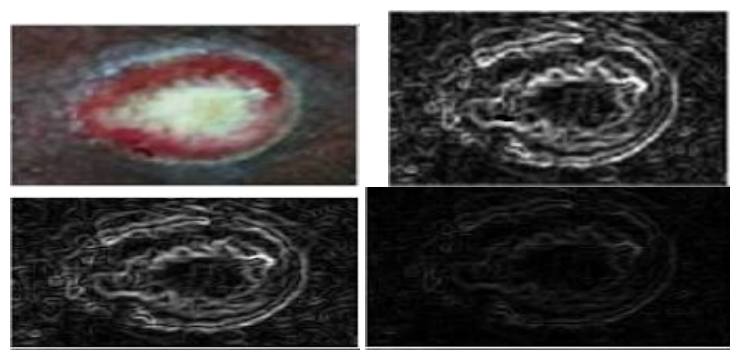

Proposed Result

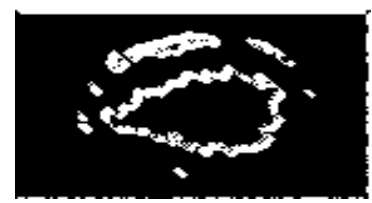

Fig 13. a) original Mycosis Fungoides Skin image

b) Edges detected by sobel operator for Mycosis Fungoides Skin image

c) Edges detected by prewitt operator for Mycosis Fungoides Skin image

d) Edges detected by robert operator for Mycosis Fungoides Skin image

e) Edges detected by proposed technique for Mycosis Fungoides Skin image

\section{CONCLUSION}

Based on the results of some traditional algorithms in edge detection we have seen the existing results. Then proposed algorithm used to find the better results in fields of image processing. In this paper, an important challenge is to detect the edge for most affected part of skin disease is accurately resolved. This paper describes the new technique for detecting edges using histogram equalization, zero cross detector,2D convolution which are useful to find the edges of most affected part of skin accurately.

\section{FUTURE SCOPE}

From Overall, implementation of edge detection for most effected part of skin disease image. Further work which can be carried out include the following.

- To perform the experiments on a large number of other skin disease images, and to conduct a full analysis of the observed results.

- Further study into the performance analysis of the proposed technique

An investigate into perfect edge detection whose primary aim is to get ground truth edge detection image

\section{ACKNOWLEDGMENTS}

This work is only complete due to the continuous encouragement of our esteemed Principal Dr.D.N.Kyatanvar(SRES COE,KOPARGAON).We are also greatful to Dr. C.Nagraju HOD,CSE Department, Yogi 
Vemana University and Dr. Debabala Swain Associate Professor CSE Department,CUTM,Jatni,Orissa for their continuous guidance. We are also thankful to SRES College Of Engineering, Kopargaon for providing us with support and technical guidance.

\section{REFERENCES}

[1] Huiyu Zhou,Jiahua Wu,Jianguo Zhang,"DIGITAL IMAGE PROCESSING:PART 1", Ventus Publishing ApS ISBN 978-87-7681-541-7

[2] Trucco," Edge detection",Chapt4 AND Jain et al., Chapt

[3] Rohit yadav, Ranbeer Tyagi, L.D.Malviya."Low Magnitude Edge Detection Algorithm".International Journal of Computer Applications, Volume 23-No.2,June 2011
[4] Sung KIM, "Applications of Convolution in Image Processing with MATLAB", University of Washington.

[5] Bernd Girod, "Digital Image Processing: Edge Detection", (C) 2013-2014 Stanford University

[6] Haris Papasaika-Hanusch,"Digital Image Processing Using Matlab",Institute of Geodesy and Photogrammetry, ETH Zurich.

[7] Gonzalez and Woods, 2008. Digital Image Processing, Third Edition, Pearson Education.

[8] P. Thakare (2011) "A Study of Image Segmentation and Edge Detection Techniques", International Journal on Computer Science and Engineering, Vol 3,No.2, 899904 